\title{
BMJ Open Towards HCV elimination among people who inject drugs in Hai Phong, Vietnam: study protocol for an effectiveness-implementation trial evaluating an integrated model of $\mathrm{HCV}$ care (DRIVE-C: DRug use \& Infections in ViEtnam-hepatitis C)
}

Delphine Rapoud (D) , ${ }^{1}$ Catherine Quillet, ${ }^{1}$ Khue Pham Minh, ${ }^{2}$ Vinh Vu Hai, ${ }^{3}$ Binh Nguyen Thanh, ${ }^{2}$ Thanh Nham Thi Tuyet, ${ }^{4}$ Hong Tran Thi, ${ }^{2}$ Jean-Pierre Molès, ${ }^{1}$ Roselyne Vallo, ${ }^{1}$ Laurent Michel, ${ }^{5}$ Jonathan Feelemyer, ${ }^{6}$ Laurence Weiss, ${ }^{7}$ Maud Lemoine, ${ }^{8}$ Peter Vickerman, ${ }^{9}$ Hannah Fraser, ${ }^{9}$ Huong Duong Thi, ${ }^{2}$ Oanh Khuat Thi Hai, ${ }^{4}$ Don Des Jarlais, ${ }^{6}$ Nicolas Nagot, ${ }^{1}$ Didier Laureillard, ${ }^{10}$ On behalf of the DRIVE-C Study Group

To cite: Rapoud D, Quillet C, Pham Minh K, et al. Towards HCV elimination among people who inject drugs in Hai Phong, Vietnam: study protocol for an effectiveness-implementation trial evaluating an integrated model of HCV care (DRIVE-C: DRug use \& Infections in ViEtnam-hepatitis C). BMJ Open 2020;10:e039234. doi:10.1136/ bmjopen-2020-039234

- Prepublication history and additional material for this paper is available online. To view these files, please visit the journal online (http://dx.doi.org/10. 1136/bmjopen-2020-039234)

DR and $C Q$ contributed equally.

Received 08 April 2020

Revised 27 August 2020

Accepted 01 October 2020

Check for updates

(C) Author(s) (or their employer(s)) 2020. Re-use permitted under CC BY-NC. No commercial re-use. See rights and permissions. Published by BMJ.

For numbered affiliations see end of article.

Correspondence to Dr Delphine Rapoud; delphine.rapoud@inserm.fr

\section{ABSTRACT}

Introduction In Vietnam, people who inject drugs (PWID), who are the major population infected by hepatitis $C$ virus (HCV), remain largely undiagnosed and unlinked to HCV prevention and care despite recommended universal hepatitis $\mathrm{C}$ treatment. The data on the outcomes of $\mathrm{HCV}$ treatment among PWID also remain limited in resourcelimited settings. The DRug use \& Infections in ViEtnamhepatitis C (DRIVE-C) study examines the effectiveness of a model of hepatitis $\mathrm{C}$ screening and integrated care targeting PWID that largely uses community-based organisations (CBO) in Hai Phong, Vietnam. In a wider perspective, this model may have the potential to eliminate HCV among PWID in this city.

Methods and analysis The model of care comprises large community-based mass screening, simplified treatment with direct-acting antivirals (DAAs) and major involvement of CBO for PWID reaching out, linkage to care, treatment adherence and prevention of reinfection. The effectiveness of DAA care strategy among PWID, the potential obstacles to widespread implementation and its impact at population level will be assessed. A cost-effectiveness analysis is planned to further inform policy-makers. The enrolment target is 1050 PWID, recruited from the DRIVE study in Hai Phong. After initiation of pan-genotypic treatment consisting of sofosbuvir and daclatasvir administrated for 12 weeks, with ribavirin added in cases of cirrhosis, participants are followed-up for 48 weeks. The primary outcome is the proportion of patients with sustained virological response at week 48 , that will be compared with a theoretical expected rate of $70 \%$.

Ethics and dissemination The study was approved by Haiphong University of Medicine and Pharmacy's Ethics

\section{Strengths and limitations of this study}

- This is the first research evaluating the efficacy of a simplified hepatitis $\mathrm{C}$ virus (HCV) care model targeting people who inject drugs (PWID) in Vietnam.

- This study relies on a strong involvement of community-based organisations at all stages of the care process.

- Patient care is carried out within public hospitals.

- Study results will provide policy-makers with key data for scaling-up HCV care to eliminate HCV transmission among PWID.

- The current high price of direct-acting antivirals in Vietnam could jeopardise widespread access to treatment.

Review Board and the Vietnamese Ministry of Health. The sponsor and the investigators are committed to conducting this study in accordance with ethics principles contained in the World Medical Association's Declaration of Helsinki (Ethical Principles for Medical Research Involving Human Subjects). Informed consent is obtained before study enrolment. The data are anonymised and stored in a secure database. The study is ongoing. Results will be presented at international conferences and submitted to international peer-review journals.

Trial registration number NCT03537196.

\section{INTRODUCTION}

Treatment of hepatitis $\mathrm{C}$ was recently revolutionised with the development of directacting antivirals (DAAs). ${ }^{1}$ Cure rate over $90 \%$ 
can be achieved with shortened treatment duration (8-12 weeks) for almost all individuals with hepatitis C. ${ }^{2}$ This progress opens a new era in which virtually all infected individuals can be cured if they are widely screened and have access to therapy. Hepatitis $\mathrm{C}$ elimination has been incorporated in the 2030 agenda of the WHO, which has set up ambitious targets as reducing new hepatitis $\mathrm{C}$ virus (HCV) infections by $80 \%$, the number of HCV-related deaths by $65 \%$, increasing hepatitis $\mathrm{C}$ diagnoses from $20 \%$ to $90 \%$ and eligible people receiving HCV treatment from $<5 \%$ to $80 \%$. $^{3}$

However, more than $80 \%$ of the hepatitis $\mathrm{C}$ burden worldwide is in low-income and middle-income countries (LMIC), where access to diagnosis and treatment is limited. ${ }^{4}$ Furthermore, people who inject drugs (PWID) are a major population affected by HCV with an estimated prevalence of approximately $60 \%-80 \% .^{5}$ WHO's objectives will not be achieved without implementing simplified and cost-effective strategies targeting key populations, in particular PWID in LMIC. While high coverage of medication-assisted treatment (MAT) and needle/ syringe exchange programmes alone cannot markedly reduce HCV transmission to low levels (unlike for HIV), new DAAs achieving high cure rate have such a potential impact. ${ }^{6}$

In Vietnam, around 1 million individuals are infected with HCV, and genotypes 1 and 6 represent almost $95 \%$ of hepatitis $\mathrm{C}$ infections. ${ }^{7-9}$ For the last few years, the strong mobilisation of civil society has been crucial to recognising hepatitis $\mathrm{C}$ as a major public health issue, and to advocating for access to treatment. Although diagnosis, treatment, including DAAs since 2018, and monitoring costs are covered partially by the national social health insurance, the remaining cost is still too high for the most vulnerable patients, including PWID. In addition, limited access to HCV genotyping represents a major obstacle for scaling-up HCV treatment.

HCV prevalence ranges from $46 \%$ to $87 \%$ among PWID in Vietnam. ${ }^{10-14}$ In 2008, local authorities piloted a new strategy policy for PWID based on access to MAT, universal antiretroviral treatment (ART) for HIV-infected PWID and a network of community-based organisations (CBO) to deliver harm reduction and distribute free syringes. Unlike HIV prevalence that has been reduced to $30 \%$ among PWID, the HCV epidemic is still very dynamic. In the DRIVE-IN (DRug use and Infections in ViEtnam - INitial phase) study (NCT02573948) conducted in 2014-2015 among PWID in Hai Phong, ${ }^{15} 16$ HCV seroprevalence was $66 \%$, $91 \%$ of HIV-infected participants were coinfected with HCV and HCV incidence was 18.8/100 person-years (95\% CI: 11.2 to 29.8$){ }^{17}$

\section{Drive program in Hai Phong}

The city of Hai Phong, with about 9000 PWID out of a population of 2 million inhabitants, has a past HIV epidemic driven by heroin injection. HIV prevalence among PWID there peaked at $60 \%$ in $2006 .{ }^{18}$ Our research group has been working in the field of drug use and HIV/
HCV infection for the past 6 years in Hai Phong. After the successful implementation of the feasibility study DRIVE-IN, the DRIVE consortium designed an innovative strategy, aiming at 'ending the HIV epidemic among PWID'. In the DRIVE study (NCT03526939), the intervention strategy includes (1) a mass screening tool (3 repeated large-scale respondent-driven sampling (RDS) surveys over 2 years) to identify HIV-infected PWID in the community, (2) linkage to HIV care (to achieve viral suppression) by strong peer support and (3) improvement of harm reduction coverage by assisting actively injecting PWID to access MAT at existing centres and to reduce unsafe injection practices. In addition to the 3 RDS surveys, 2 cohorts were implemented, with follow-up (FU) visits at study sites every 6 months: (1) among HIV positive to monitor the intervention implementation and identify potential issues in the cascade of care and (2) among HIV negative to monitor HIV incidence and risk factors. The third RDS survey (DRIVE RDS3) was launched in October 2018.

\section{Research hypothesis}

The DRIVE study offers a unique opportunity to extend interventions to hepatitis C. We hypothesise that a simplified model addressing all the following challenges could lead, if scaled up, to the elimination of HCV among PWID in Hai Phong:

- Identification of PWID with hepatitis C: to date only HIV-infected PWID registered in HIV outpatient clinics or PWID registered in methadone treatment centres have access to HCV screening with serology testing. HCV RNA is not routinely available.

- Improving health-seeking behaviours and referral to HCV care: PWID often have poor linkage with healthcare system, including fear or experience of stigmatisation, lack of self-esteem, limited access to health insurance and marginalisation.

- Setting-up a model of HCV care integrated in the local healthcare system: authorities will not launch specific HCV infection programmes but rather will integrate $\mathrm{HCV}$ care and treatment within existing infectious diseases or hepatology departments of health facilities.

- Optimised and tailored support for treatment adherence: some PWID present characteristics that may hamper medicine adherence (psychiatric disorders and marginalisation).

- Prevention of HCV reinfection after cure, which is crucial in a context of high HCV incidence: specific harm reduction and counselling activities, other than those for HIV, need to be elaborated.

\section{METHODS AND ANALYSIS Objectives}

The primary objective of DRug use \& Infections in ViEtnam-hepatitis C (DRIVE-C) is to assess the effectiveness of the proposed model of hepatitis $\mathrm{C}$ communitybased screening and integrated care targeting PWID in Hai Phong, Vietnam. 
Secondary objectives include: to assess all steps of the hepatitis $\mathrm{C}$ cascade of care; to assess the occurrence of adverse events and drug-related side effects; to evaluate adherence to HCV treatment; to determine factors associated with treatment failure; to estimate the reinfection rate at the end of the study; to identify risk factors for $\mathrm{HCV}$ reinfection and to project the impact and cost effectiveness of the implemented HCV treatment intervention.

\section{Outcome measures}

The primary endpoint is the proportion of all patients in success of the model of care, defined by HCV RNA $<10$ $\mathrm{IU} / \mathrm{mL}$ at the end of the study (week 48 visit).

What will be considered as failures: (1) detectable HCV RNA at "sustained virological response at post-treatment week 12" (SVR12) visit or at the end of study visit (HCV RNA $\geq 10 \mathrm{IU} / \mathrm{mL}$ ), (2) missing HCV RNA result at the end of study visit, (3) HCV treatment not initiated within 1 year of HCV screening, (4) permanent discontinuation of DAAs, (5) death and (6) discontinuation of the study due to loss to FU or transfer out.

Secondary endpoints include the following:

- Evaluation of the HCV cascade of care, among all those with hepatitis $\mathrm{C}$ infection.

- Reinfection rate defined by HCV RNA $\geq 10 \mathrm{IU} / \mathrm{mL}$ at the end of the study among cured participants (HCV $\mathrm{RNA}<10 \mathrm{IU} / \mathrm{mL}$ at the SVR12 visit).

- Mortality rate among all participants.

- Frequency, type and time to grade 3 or 4 , adverse clinical or biological events.

- Frequency, type and time to drug-related clinical or biological adverse reactions of grade 3 or 4 or leading to treatment interruption.

- Adherence assessed by self-questionnaire on DAA drug intake and drug accountability for DAAs.

- Factors associated with HCV treatment failure or HCV reinfection.

- Effect of the HCV treatment intervention on HCV infections and disability-adjusted life years (DALYs) averted, quality-adjusted life years (QALYs) saved, HCV incidence and prevalence as projected by the model under various scenarios.

- Incremental cost-effectiveness ratio (ICER).

\section{Study design}

Because the aim of this research is to demonstrate how known effective treatment can be used in the Vietnamese context, we will conduct an effectiveness-implementation hybrid study type $1 .{ }^{19}$ In addition, a modelling exercise to assess the impact of the intervention at the population level and a cost-effectiveness analysis to further inform policy-makers are included in the study.

The strategy of the model includes:

- Mass detection of hepatitis C infection among PWID, through a large community-based RDS survey.

- Community-based support to improve referral to specific care for those identified with hepatitis C infection.
- HCV care delivery integrated within the existing health system, with a simplified treatment protocol based on a combination of DAAs and considering PWID factors, such as frequent HIV co-infection and methadone treatment.

- Optimised treatment adherence through a combination of healthcare therapeutic education and CBO support.

- Increase in harm reduction activities to encompass HCV transmission risk and to prevent HCV reinfection.

Patients will be followed for 48 weeks after initiating HCV treatment.

\section{Study population}

Participants enrolled are PWID who (1) either participated in the DRIVE RDS3 survey or have been enrolled in the DRIVE HIV-positive or HIV-negative cohort and performed an FU visit after RDS3, (2) have a hepatitis C infection defined by a positive HCV RNA at the time of screening and (3) sign the informed consent form.

All DRIVE participants were enrolled through RDS surveys and were active PWID, defined by a positive urine test result for heroin and recent injection marks, at the time of enrolment in DRIVE. At the time of enrolment in DRIVE-C, some of them may no longer inject drugs.

Non-inclusion criteria include: severe associated diseases requiring specific treatment; any condition which might compromise the safety of the patient by participating in the study; previous history of DAA use; contraindication to sofosbuvir or daclatasvir; for women of childbearing potential: pregnancy, breastfeeding or refusal to use a contraceptive method; renal failure with creatinine clearance $\leq 30 \mathrm{~mL} / \mathrm{min}$; being deprived of freedom by a judicial or administrative decision; planning to move out of Hai Phong in the next 12 months and being unable to understand the study.

\section{Study schedule}

The total duration of the study will be 3 years, including 6 months of preparation, 1 year for enrolling and starting HCV treatment for all participants, 1 year of FU for each participant, and 6 months of data analysis.

\section{Study settings}

Hepatitis C screening takes place in two DRIVE study sites run by CBO. Enrolment into DRIVE-C and FU are conducted in hepatitis clinics integrated within the departments of infectious and tropical diseases of three geographically distributed hospitals throughout the city (Viet Tiep Hospital, Kien An Hospital and Thuy Nguyen Hospital), representing both district and provincial level care settings.

\section{Implementation}

\section{Screening}

During DRIVE RDS surveys and cohort FU visits, participants are tested for HCV antibodies using rapid diagnostic test (SD Bioline) at the study community sites. At 
the time of DRIVE RDS3 survey or a DRIVE cohort FU visit, participants (1) known to have an HCV seropositive result from a previous DRIVE visit or (2) newly screened HCV seropositive at that visit are informed of the nested research programme on hepatitis $\mathrm{C}$ infection (DRIVE-C). On participant's agreement, a blood sample is sent to a centralised laboratory, where the diagnosis of chronic hepatitis C is done using the GeneXpert System (Xpert HCV Viral Load test from Cepheid). Result is provided within 10 days.

Participants with hepatitis $\mathrm{C}$ infection proven by positive HCV RNA $(\geq 10 \mathrm{IU} / \mathrm{mL})$ are proposed to enrol in DRIVE-C. If interested, they will be contacted by CBO members to schedule an appointment at one of the three hepatitis clinics.

\section{Enrolment and FU}

At the pre-inclusion visit, the physician checks that the participant fulfils the inclusion criteria and does not meet any of the non-inclusion criteria, then proceeds to the informed consent process. After consent obtention, clinical, laboratory and imaging assessments are performed. Liver assessment is based on the calculation of aspartate transaminase (AST) to Platelet Ratio Index score, abdominal ultrasound and fasting liver stiffness measurement with Fibroscan (Echosens). ART initiation and/or hepatitis $B$ virus (HBV) treatment initiation are prescribed first when necessary. For those who are not already receiving methadone maintenance treatment (MMT), MMT initiation is strongly recommended.

The patient is successfully enrolled if all criteria are confirmed at the inclusion visit, planned as far as possible not later than 2 weeks after the pre-inclusion visit. After DAA initiation at inclusion visit, participants will undergo seven FU visits until the end of study (table 1 and figure 1). They shall include clinical examination, biological testing, drug dispensation, therapeutic education, assessment of adherence, referral to appropriate care in case of an ongoing adverse event, HCV-related counselling session focusing on reinfection after the end of treatment, administration of questionnaires and blood collection for sample repository.

End of treatment visit is performed 12 weeks or 24 weeks after treatment initiation according to its duration.

SVR12 visit and end of study visit are performed 12 weeks after the end of treatment and 48 weeks after treatment initiation, respectively, and include both HCV RNA testing and HCV genotyping in case of detectable HCV RNA.

In the interval between two scheduled visits, patients have access to medical personnel whenever they become ill.

\section{Drugs}

In accordance with WHO and Vietnamese guidelines, the standard HCV treatment in DRIVE-C is sofosbuvir $400 \mathrm{mg}$ /day and daclatasvir $60 \mathrm{mg} /$ day for 12 weeks. The choice of DAA regimen was based on our willingness to have a pan-genotypic regimen and to use drugs already registered in Vietnam, to allow quick scale up of the model if effective, while taking into account the potential interactions with ART for co-infected patients.

For HIV-infected participants receiving efavirenz or nevirapine, daclatasvir dose is increased to $90 \mathrm{mg}$ /day. In case of cirrhosis, ribavirin is added to sofosbuvir/ daclatasvir during the 12 weeks of treatment. Cirrhosis is defined by a Fibroscan value $>12.5 \mathrm{kPa}^{20}{ }^{21}$ In case of ribavirin contraindication or side effects leading to ribavirin discontinuation, sofosbuvir/daclatasvir combination is prescribed for 24 weeks. Sofosbuvir and daclatasvir are purchased from Hetero Drugs, a generic manufacturer granted by Gilead and BMS companies. Drugs are prescribed by study doctors and delivered by hospital pharmacists.

Patients receiving ART and/or MMT will start HCV treatment after at least 3 months of ART and 2 weeks of MMT. All participants are tested for hepatitis B surface antigen (HBsAg) and hepatitis B surface antibody (HBsAb) at preinclusion visit. Participants with positive HBsAg already treated initiate HCV treatment without delay. Participants with positive HBsAg not yet treated will be first treated for HBV. An appointment for HCV treatment initiation is provided within 1 month after $\mathrm{HBV}$ treatment initiation. Participants with negative HBsAg and $\mathrm{HBsAb}$ at preinclusion visit and who experience an elevation of ALT $>5$ times upper normal limit during $\mathrm{HCV}$ treatment FU will be retested for HBsAg and, if negative, tested for hepatitis B core antibody (HBcAb). In case of HBsAg or HBcAb positive, HBV DNA is measured to detect possible HBV flare-ups or reactivation as recommended by the WHO. Tenofovir will be introduced in case of detectable HBV DNA. HBV immunisation is recommended to participants with negative $\mathrm{HBsAg}$ and negative HBsAb.

\section{Role of peer educators}

Seven CBO, coordinated by the Vietnamese nongovernmental organisation Supporting Community Development Initiatives, are involved in DRIVE-C. CBO members both intervene (1) in the three hepatitis clinics, where they provide counselling and conduct questionnaires, and (2) in the field where each $\mathrm{CBO}$ member is assigned participants to follow and support on a regular basis to overcome potential obstacles regarding: access to MMT and/or ART, adherence to HCV treatment, administrative tasks and access to harm reduction materials. They are also in charge of counselling and tracing participants for missed study visits.

\section{Data collection and data management}

The data collected are recorded in an electronic case report form (CRF), using the Ennov Clinical software accredited by the US Food and Drug Administration (FDA). They include: sociodemographic information, medical history, clinical evaluation, laboratory test and imaging results, drug uptake, adverse events, social events, 


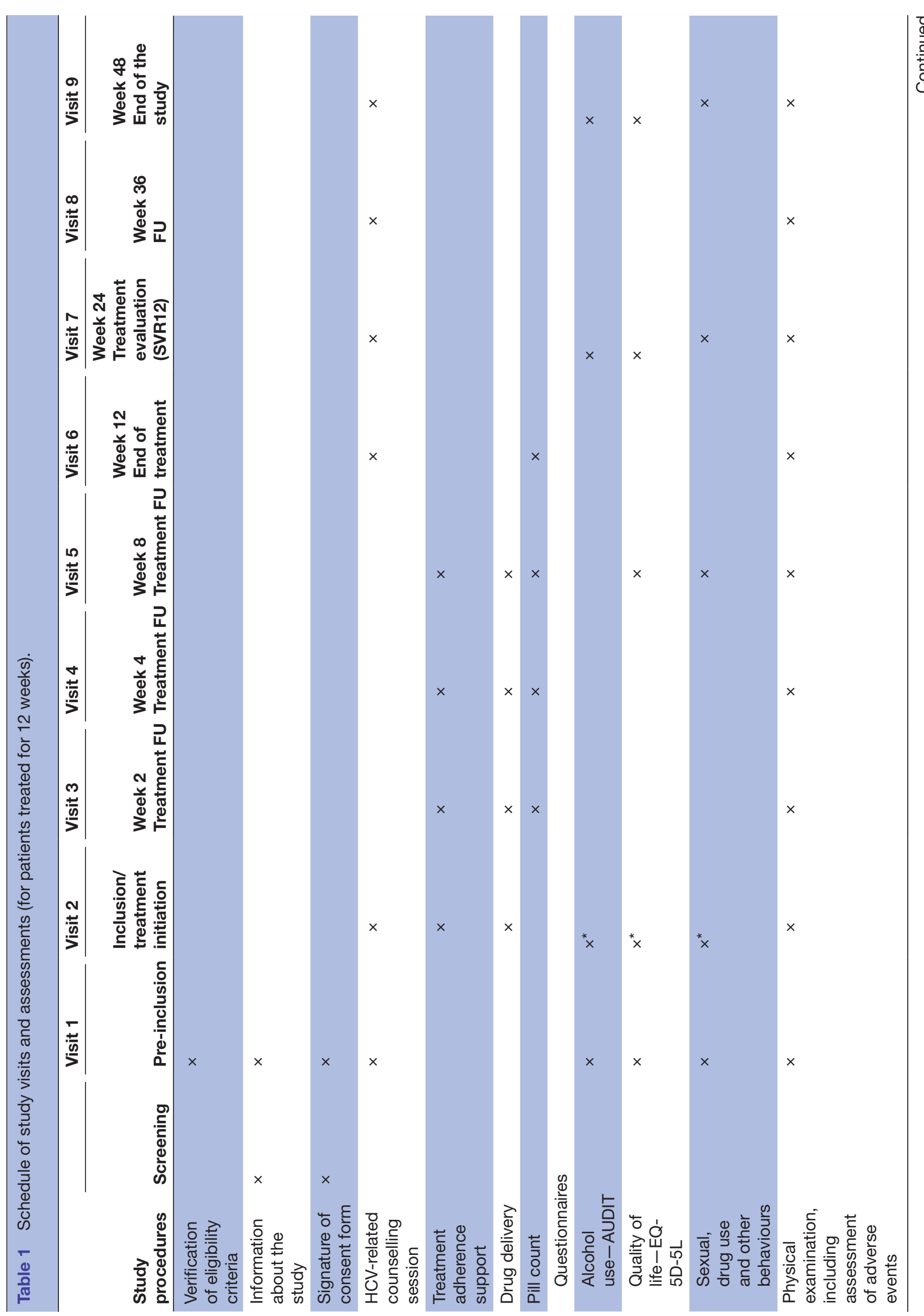




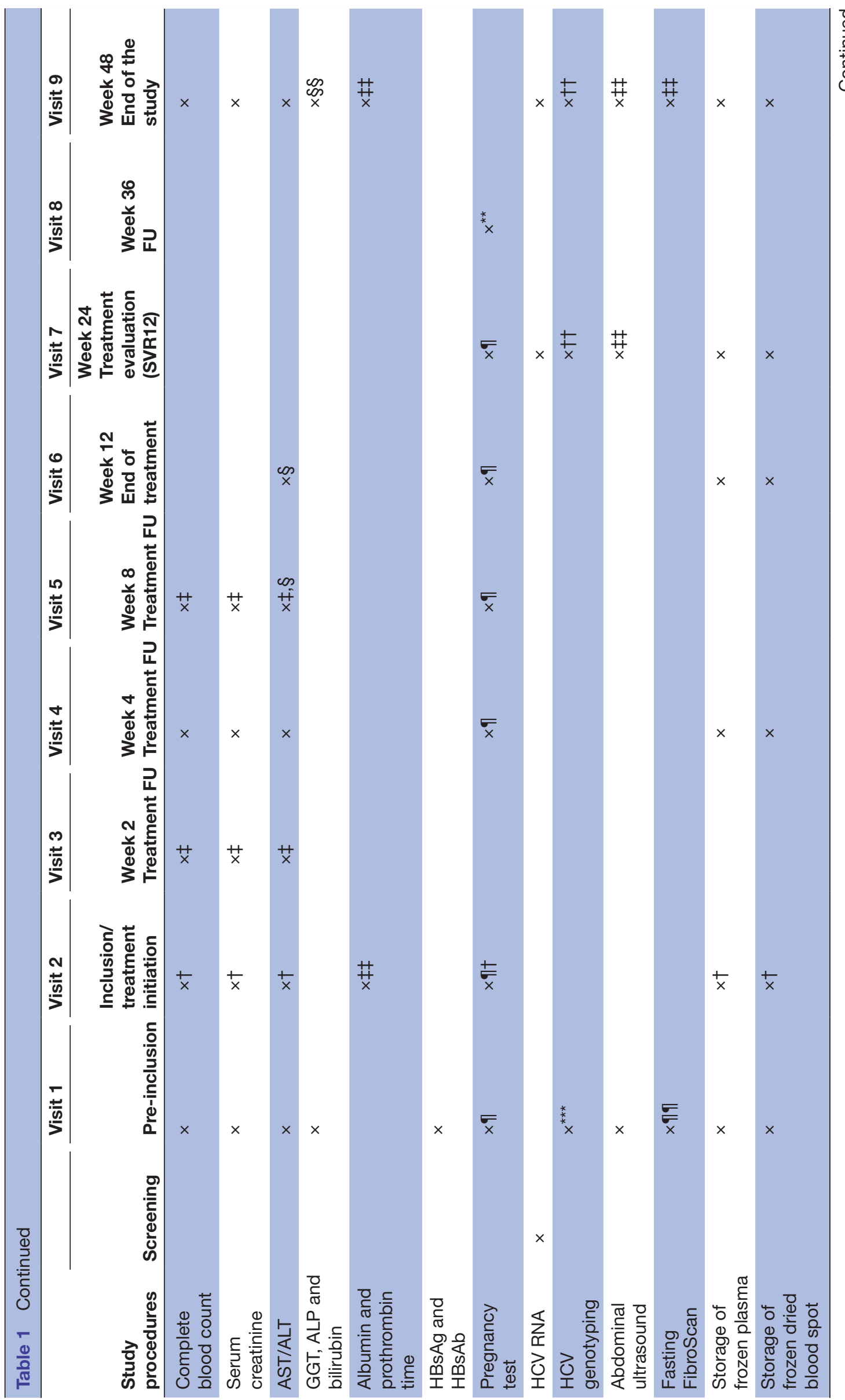

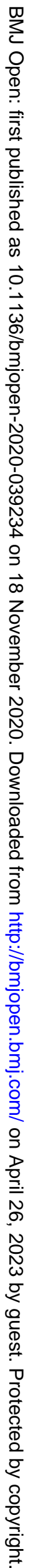




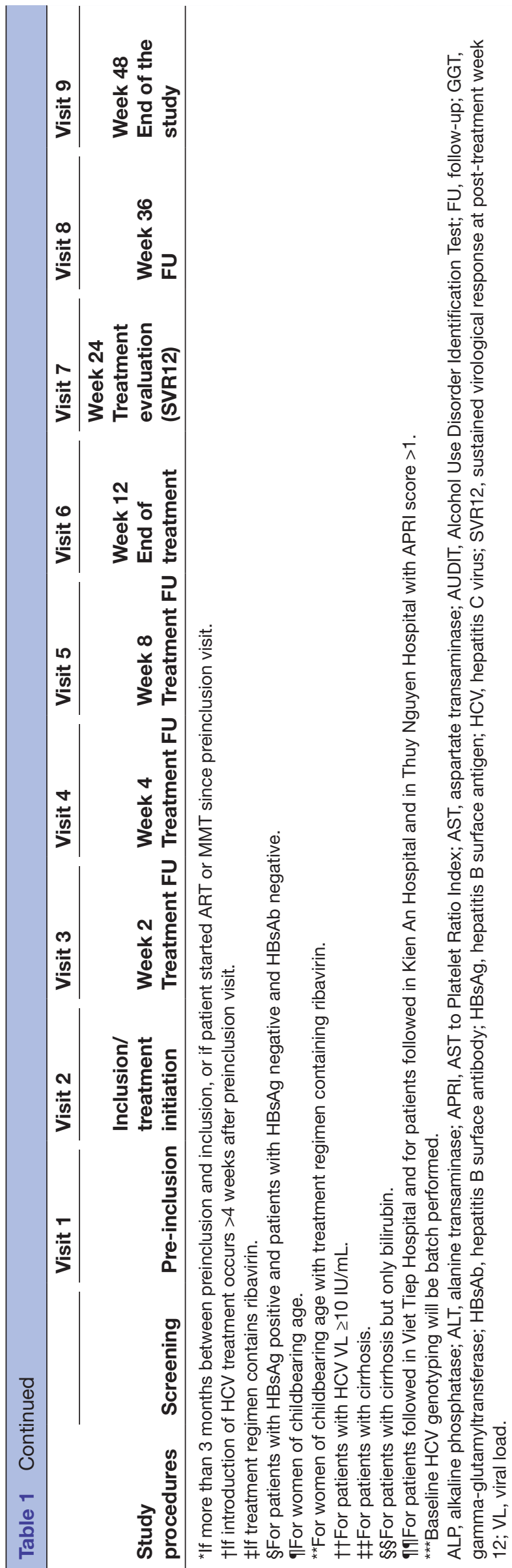

including incarceration, alcohol use: Alcohol Use Disorder Identification Test, quality of life: EQ-5D (EuroQol-5D), ${ }^{22}$ sexual, drug use and other behaviours selfreported by participants and focusing on HCV infection or reinfection risks.

Verification of the completeness and consistency of the data is performed according to a specific data validation plan. Data management is performed both at the international coordinating trial unit (CTU) level (Pathogenesis and Control of Chronic Infections, UMR 1058-INSERM, Univ Montpellier, EFS, Montpellier, France) and at the local CTU level when feasible (Faculty of Public Health, Haiphong University of Medicine and Pharmacy, Hai Phong, Vietnam). Centralised queries are sent by the international CTU to the local CTU and by the local CTU to study clinical sites under the terms of the verification defined in the data validation plan.

\section{Safety reporting}

All biological and clinical adverse events (AE) are reported in a specific CRF AE form, and all serious adverse events are notified to the sponsor, who is responsible for the assessment of their causality in relation to the study drugs, concomitant medication and the research.

\section{Sample size}

Power calculation for the model of care assessment

DRIVE RDS3 survey aims to enrol 1500 participants. According to DRIVE first and second rounds of RDS data, we expect the number of participants at each step as described in figure 2. These numbers will provide us with a precise estimation of outcomes. The proportion of PWID with HCV chronic infection who are cured is expected to be $90 \% \times 90 \% \times 90 \%=73 \%$. The enrolment of about 900 PWID with HCV chronic infection will allow estimating the expected cure rate of $73 \%$ with a precision of $\pm 2.9 \%$. With a lower cure rate of $50 \%$, we would have a similar precision of $\pm 3.3 \%$.

According to DRIVE RDS2 and FU data, an estimated 567 cohort participants, not having attended the RDS3 will be screened for hepatitis at cohort FU visits.

A total of 1050 patients will be included in the study. This number of patients will allow us to explore the treatment-response heterogeneity, partly through subgroup analyses based on key characteristics, such as HIV co-infection, liver fibrosis stage or genotypes. Finally, enrolling all HCV-infected individuals identified in one mass screening round (RDS) as a unit will allow the mathematical model to estimate to what extent the repetition of RDS could tackle HCV transmission in Hai Phong.

Power calculation for the reinfection rate

Among the $650+295=945$ patients cured (SVR12), we estimate that $90 \%$ (850) will be assessed for reinfection at week 48 . Based on these assumptions and setting the alpha risk to $5 \%$, we will be able to estimate an expected reinfection rate of $3 \%$ with a precision of $\pm 1.3 \%$, or an expected reinfection rate of $5 \%$ with a precision of $\pm 1.6 \%$. 


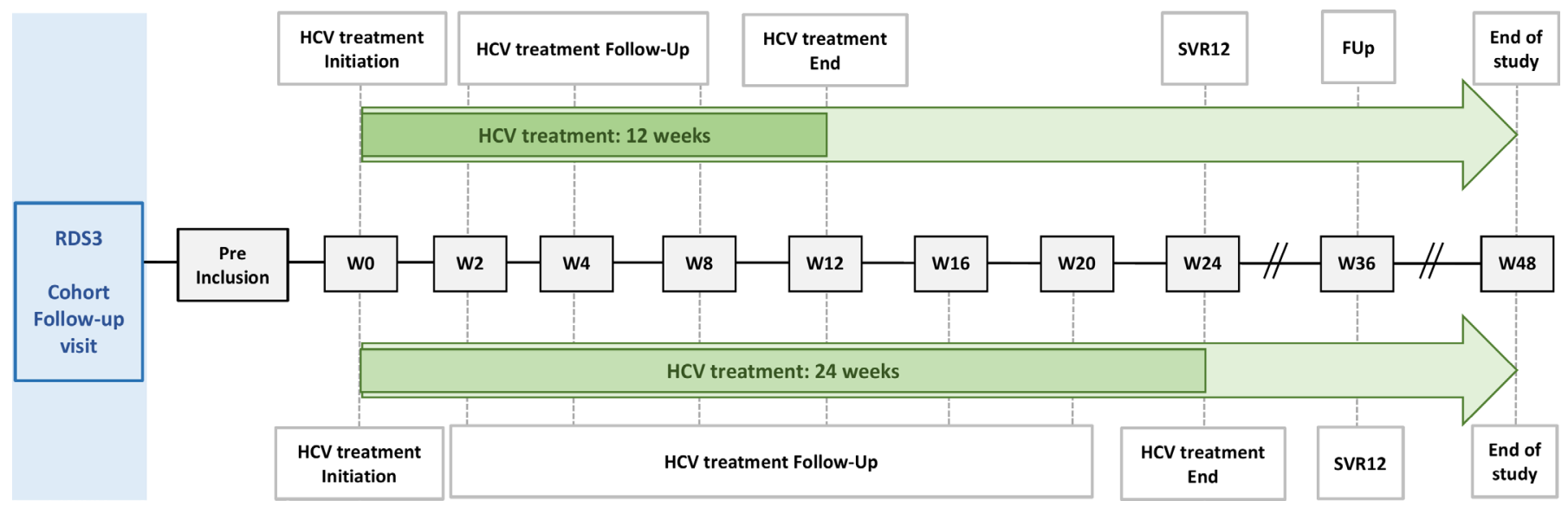

Figure 1 Patient visit schedule flowchart. HCV, hepatitis C virus; FUp, follow-up; RDS, respondent-driven sampling; W0, week 0 ; SVR12, sustained virological response at post-treatment week 12.

\section{Analysis strategies}

For the intention to treat analysis, the model of HCV care will be analysed separately for the DRIVE RDS3 and for cohort FU visits participants. The latter will reflect the ability to treat and cure all the HCV-infected patients who are already in HIV and addiction care. For each analysis, all participants will be included in the analysis, whatever their FU (including patients who died, were lost to FU, or withdrew from the study). For the on-treatment analysis, we will consider only patients who started HCV treatment.

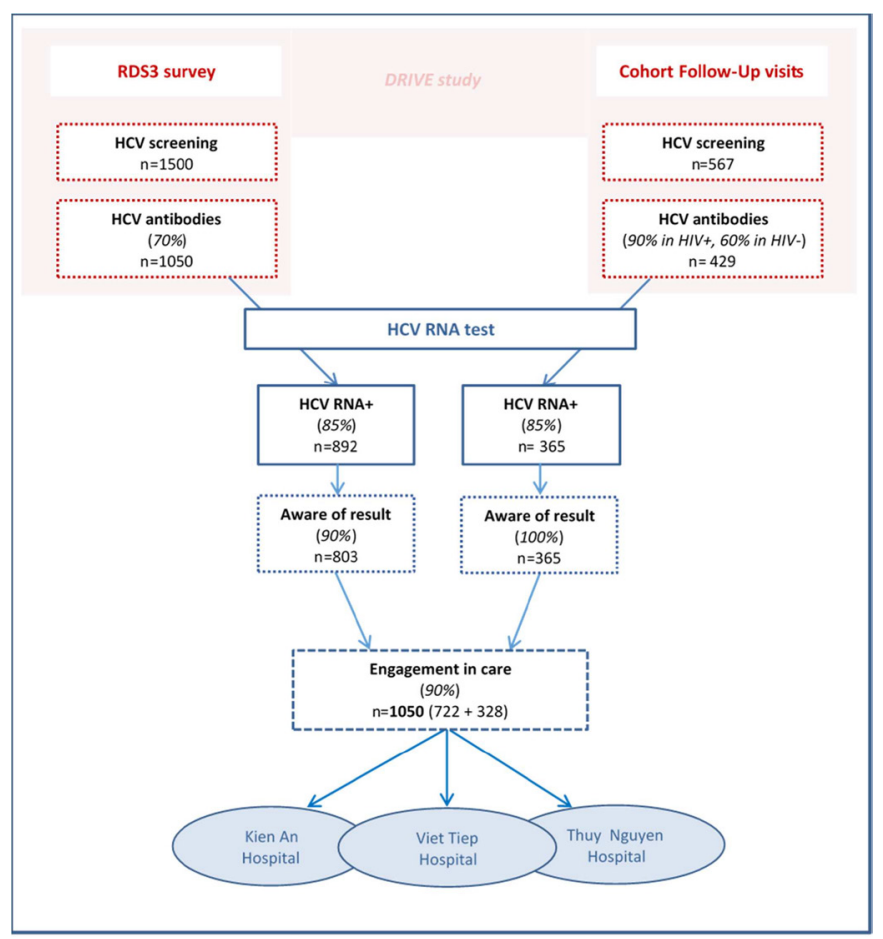

Figure 2 Study pre-enrolment diagram. HCV, hepatitis C virus; RDS3, third respondent-driven sampling.

\section{Statistical methods}

A detailed plan of analysis will be elaborated and validated by the Scientific Advisory Board (SAB) before the end of enrolment.

The proportion of patients who have met the primary outcome (ie, plasma HCV RNA below $10 \mathrm{IU} / \mathrm{mL}$ at week 48) will be calculated with its $95 \%$ CI. The primary outcome will be compared with a theoretical value of $70 \%$ (rounded from 73\%) using a classic one-sided test based on an approximation by the normal distribution. This value represents the expected rate of patients with chronic HCV infection who will be cured. The primary outcome will then be calculated in subgroups, such as gender or type of drug use.

All secondary outcomes will be described with their 95\% CI. We will then investigate the treatment-response heterogeneity, whereby some PWID may adhere or respond differently to the treatment because of measurable characteristics. For this purpose, we will use a logistic regression model to identify the factors associated with failure to cure, as well as subgroup analyses (including gender and HIV infection) to assess the cure rate variations across the population characteristics.

The approaches to deal with missing data will be described in the detailed plan of analysis. In brief, the primary and secondary outcomes will be evaluated according to a complete case analysis. In order to assess the factors associated with outcomes failures, we will use a multiple imputation approach to replace missing data when they represent more than $1 \%$ of all data for a given variable.

\section{Modelling and cost-effectiveness analysis}

Mathematical modelling, developed by a team from Department of Population Health Sciences at the University of Bristol, the United Kingdom, will be used to estimate the impact and cost effectiveness of scaling up the HCV care model, compared with the baseline level of prevention and treatment. The model will be adapted from an existing open dynamic, deterministic model 
of HCV transmission, progression and HCV treatment among PWID and ex-PWID communities. ${ }^{18} 2324$ The model will be fit within a Bayesian framework to epidemiological, behavioural and intervention related data from the DRIVE and DRIVE-C study. Crucially, the model will be able to account for the 'prevention benefit' of treatment as well as reduction in HCV-related morbidity. The impact modelling will consider if the intervention could reduce HCV transmission to low levels $(90 \%$ reduction in HCV incidence and mortality) among PWID in the next $5-15$ years, as set out by the recent WHO elimination targets. ${ }^{3}$ Expenditure data will be collected from the DRIVE-C intervention with direct and indirect costs allocated to activities, such as diagnosis, baseline assessment, treatment initiation and FU visits. Staff costs will be assigned to activities based on average length of visits using time sheets completed by patient-facing personnel. Unit costs for laboratory tests and medicines will be taken from invoices or price lists where relevant. The cost per patient will be calculated using resource use data from the study for each patient tested or treated for HCV. Quality of life data (EQ-5D-5L) ${ }^{22}$ will be collected as part of the study to estimate changes in utility due to treatment. The cost-effectiveness analysis will estimate the mean ICER in terms of cost per QALY saved, which will be compared against standard thresholds for interventions being cost effective in LMIC. ${ }^{25} 26$

\section{Patient and public involvement}

CBO members, as peer educators and, for those infected with $\mathrm{HCV}$, as patients' representatives, have been involved since the very beginning in the process of study design and implementation through brainstorming sessions, including identifying the research questions. They provided an insight into patients' needs and the currently existing gaps in the health system regarding the specific population of PWID. They took part in elaborating the procedures, the information notice, the consent form and other tools dedicated to patients, such as counselling materials. Through the RDS survey strategy, patients participate in recruiting other patients in the study.

\section{Study oversight}

The overall management of the study is carried out by the coordination team composed of the two co-principal investigators, and the international and local CTU.

An SAB is established to ensure the proper conduct of the trial across its scientific, methodological, and ethical aspects and the protection of participants. This SAB includes members of the coordination team, external experts and representatives of PWID.

An independent Data and Safety Monitoring Board (DSMB) will review data in order to advise the sponsor and the SAB. The DSMB monitors the main safety and efficacy outcome measures and the overall conduct of the study, with the aim of protecting the safety and the interests of the study participants. It is composed by experts in hepatitis $\mathrm{C}$ clinical management and statistics.

\section{DISCUSSION}

Although DAA drugs allow achieving very high rates of HCV cure among patients (at the individual level), we still do not know how to integrate DAA in the health system in LMIC, and whether HCV elimination can be achieved at the population level among high-risk groups, such as PWID. Our research could demonstrate that an integrated model of care, including a simplified treatment protocol accounting for comorbidities (addiction and HIV), and important support from $\mathrm{CBO}$ for linkage to care after screening, DAA adherence and prevention of reinfection after cure could lead to achieve high rate of $\mathrm{HCV}$ cure among PWID screened with chronic hepatitis C. The modelling and cost-effectiveness analyses will show to what extent this model of care, if scaled up through repeated RDS, could lead rapidly to HCV elimination and is worth the initial investment. The reinfection rate issue will not be fully addressed in this project because of the limited FU after SVR12. However, recent information suggest that this rate is the highest shortly after cure and then decreases with time. The DRIVE-C results, first research on assessing the efficacy of a model of HCV care among PWID in Vietnam, will inform decision-makers and will support replication and adaptation of the intervention to other contexts.

\section{Ethics and dissemination}

Study approval

The protocol was approved by Haiphong University of Medicine and Pharmacy's Ethics Review Board and the Vietnamese Ministry of Health.

\section{Consenting participant}

Potential participants are informed by the investigator verbally and via the information sheet of the objectives, duration, potential risks and benefits of the study, of any discomfort it may entail, and that they are free, without justification, to withdraw at any time. The investigator ensures the participant understanding and adequate answers to all his/her questions before the signature of the consent form (online supplemental file 1). The study is free of charge for participants who receive financial compensation only for the transportation costs.

\section{Confidentiality}

All data recorded in the framework of this study, including subject medical information, are strictly confidential and coded, using a unique study subject identification code. Access to participant data is restricted to investigators and appropriate study staff. Published results will not contain any personal identifying data. In case of consent withdrawal, no new information will further be collected and recorded in the database. The data collected prior to the withdrawal will be used for the analysis, unless the participant expresses the will that his/her data be removed from the database.

Care of peer educators

All CBO members involved in the DRIVE-C study and their partners are proposed to be tested for HCV. When 
diagnosed with a chronic hepatitis $\mathrm{C}$, they receive therapeutic education and free HCV treatment.

\section{Dissemination plan}

Final results are expected by mid-2021. Relevant results will be shared with participants, investigators and national authorities, disseminated through peer-review international journals and presented at national and international conferences, as well as community-based events organised in Vietnam.

\section{Trial status}

PWID eligible for DRIVE-C were identified from October 2018 to May 2019, and enrolment in the study started in November 2018. Among 1201 eligible participants, 1022 were referred to hepatitis clinics. HCV treatment initiation and $\mathrm{FU}$ are ongoing.

\section{Author affiliations}

${ }^{1}$ Pathogenesis and Control of Chronic Infections, Inserm, Etablissement Français du Sang, University of Montpellier, Montpellier, France

${ }^{2}$ Faculty of Public Health, Haiphong University of Medicine and Pharmacy, Hai Phong, Vietnam

${ }^{3}$ Department of Infectious and Tropical Diseases, Viet Tiep Hospital, Hai Phong, Vietnam

${ }^{4}$ Center for Supporting Community Development Initiatives, Hanoi, Vietnam ${ }^{5}$ CESP Inserm UMRS 1018, Paris Saclay University, Pierre Nicole Center, French Red Cross, Paris, France

${ }^{6}$ College of Global Public Health, New York University, New York, New York, USA ${ }^{7}$ Department of Clinical Immunology, Hôtel Dieu Hospital, Paris, France

${ }^{8}$ Department of Surgery and Cancer, Liver Unit, Imperial College London, London, UK ${ }^{9}$ Population Health Sciences, Bristol Medical School, University of Bristol, Bristol, UK ${ }^{10}$ Department of Infectious and Tropical Diseases, Nîmes University Hospital, Nîmes, France

Acknowledgements The DRug use \& Infections in ViEtnam-hepatitis C (DRIVE-C) study group acknowledge people who inject drugs for their participation in the study. We are indebted to members of the seven community-based organisations (Friendship Arms, Lighthouse, Lotus, White Sand, Virgin Flowers, Sunrise and An Duong Sun), to the Molecular Biology Laboratory of Haiphong University of Medicine and Pharmacy (Dr Bach Thi Nhu Quynh) and to health professionals of the three hepatitis clinics in Kien An hospital, Thuy Nguyen hospital and Viet Tiep hospital, for their involvement to make this study possible. We are grateful to ANRS for funding. We thank Claire Rekacewicz, Isabelle Fournier, Maria-Camila Calvo Cortes and Joséphine Balssa for their active support at ANRS headquarters. We thank Hai Phong's health authorities, Haiphong University of Medicine and Pharmacy leaders and the three hospital directors for their strong support since the beginning of the collaboration. We thank the DRIVE-C's Scientific Advisory Board chaired by Karine Lacombe and Duong Thi Huong for significant contribution to the study (members: Philip Bruggmann, Le Minh Giang, Nguyen Hoang Long, Pham Thi Minh, Phan Thi Thu Huong and Vu Van Cuong), and the DRIVE-C's Data and Safety Monitoring Board chaired by Stanislas Pol for careful analysis of the trial progress and helpful advice (members: Delphine Gabillard and Isabelle Andrieux-Meyer). We thank Françoise Barré-Sinoussi and Jean-François Delfraissy for continuous support and helpful advice.

Contributors KPM and DL: coordinating investigators of DRIVE-C. KPM, DL, NN, DDJ, J-PM, LM, PV, HF, LW, ML, OKTH and HDT: study conception, planning and design. KPM, DL, NN, J-PM, LM, PV, HF, OKTH, VVH, RV, CQ and DR: protocol preparation. CQ, BNT, TNTT and HTT: study management. DR and CQ: drafting of the manuscript. PV, DDJ, JF, J-PM and DL: editing the manuscript. All authors significantly contributed to the manuscript and approved the final version of the manuscript.

Funding DRug use \& Infections in ViEtnam-hepatitis C (DRIVE-C) is funded and sponsored by French National Institute for Health and Medical Research-France REcherche Nord \& sud Sida-hiv Hépatites (Inserm-ANRS), 101 rue de Tolbiac, 75013 Paris, France (ANRS 12380 DRIVE-C).
Competing interests None declared.

Patient consent for publication Not required.

Provenance and peer review Not commissioned; externally peer reviewed.

Supplemental material This content has been supplied by the author(s). It has not been vetted by BMJ Publishing Group Limited (BMJ) and may not have been peer-reviewed. Any opinions or recommendations discussed are solely those of the author(s) and are not endorsed by BMJ. BMJ disclaims all liability and responsibility arising from any reliance placed on the content. Where the content includes any translated material, BMJ does not warrant the accuracy and reliability of the translations (including but not limited to local regulations, clinical guidelines, terminology, drug names and drug dosages), and is not responsible for any error and/or omissions arising from translation and adaptation or otherwise.

Open access This is an open access article distributed in accordance with the Creative Commons Attribution Non Commercial (CC BY-NC 4.0) license, which permits others to distribute, remix, adapt, build upon this work non-commercially, and license their derivative works on different terms, provided the original work is properly cited, appropriate credit is given, any changes made indicated, and the use is non-commercial. See: http://creativecommons.org/licenses/by-nc/4.0/.

ORCID iD

Delphine Rapoud http://orcid.org/0000-0001-6677-2714

\section{REFERENCES}

1 Liang TJ, Ghany MG. Current and future therapies for hepatitis C virus infection. N Engl J Med 2013;368:1907-17.

2 Pawlotsky J-M, Feld JJ, Zeuzem S, et al. From non-A, non-B hepatitis to hepatitis $C$ virus cure. $J$ Hepatol 2015;62:S87-99.

3 WHO. Combating hepatitis B and C to reach elimination by 2030 , 2016. Available: http:/apps.who.int/iris/bitstream/10665/206453/1/ WHO_HIV_2016_eng.pdf

4 Mohd Hanafiah K, Groeger J, Flaxman AD, et al. Global epidemiology of hepatitis $C$ virus infection: new estimates of age-specific antibody to HCV seroprevalence. Hepatology 2013;57:1333-42.

5 Nelson PK, Mathers BM, Cowie B, et al. Global epidemiology of hepatitis $B$ and hepatitis $C$ in people who inject drugs: results of systematic reviews. Lancet 2011;378:571-83.

6 Martin NK, Vickerman P, Grebely J, et al. Hepatitis C virus treatment for prevention among people who inject drugs: modeling treatment scale-up in the age of direct-acting antivirals. Hepatology 2013;58:1598-609.

7 Pham DA, Leuangwutiwong P, Jittmittraphap A, et al. High prevalence of hepatitis $\mathrm{C}$ virus genotype 6 in Vietnam. Asian Pac $J$ Allergy Immunol 2009;27:153-60.

8 Pham VH, Nguyen HDP, Ho PT, et al. Very high prevalence of hepatitis $C$ virus genotype 6 variants in southern Vietnam: largescale survey based on sequence determination. Jpn J Infect Dis 2011;64:537-9.

9 Tanimoto T, Nguyen $\mathrm{HC}$, Ishizaki A, et al. Multiple routes of hepatitis $C$ virus transmission among injection drug users in HAI Phong, Northern Vietnam. J Med Virol 2010;82:1355-63.

10 Clatts MC, Colón-López V, Giang LM, et al. Prevalence and incidence of HCV infection among Vietnam heroin users with recent onset of injection. J Urban Health 2010;87:278-91.

11 Gish RG, Bui TD, Nguyen CTK, et al. Liver disease in Viet Nam: screening, surveillance, management and education: a 5-year plan and call to action. J Gastroenterol Hepatol 2012;27:238-47.

12 Kallman JB, Tran S, Arsalla A, et al. Vietnamese community screening for hepatitis B virus and hepatitis C virus. J Viral Hepat 2011;18:70-6.

13 Nakata S, Song P, Duc DD, et al. Hepatitis C and B virus infections in populations at low or high risk in Ho Chi Minh and Hanoi, Vietnam. $J$ Gastroenterol Hepatol 1994;9:416-9.

14 Nguyen VTT, McLaws M-L, Dore GJ. Prevalence and risk factors for hepatitis $C$ infection in rural North Vietnam. Hepatol Int 2007;1:387-93

15 Des Jarlais D, Duong HT, Pham Minh K, et al. Integrated respondentdriven sampling and peer support for persons who inject drugs in Haiphong, Vietnam: a case study with implications for interventions. AIDS Care 2016;28:1312-5.

16 Des Jarlais DC, Thi Huong D, Thi Hai Oanh K, et al. Prospects for ending the HIV epidemic among persons who inject drugs in Haiphong, Vietnam. Int J Drug Policy 2016;32:50-6.

17 Molès J-P, Vallo R, Khue PM, et al. HIV control programs reduce HIV incidence but not $\mathrm{HCV}$ incidence among people who inject drugs in HaiPhong, Vietnam. Sci Rep 2020;10:6999. 
18 Ministry of Health - Vietnam Administration of HIV/AIDS Control. Viet Nam HIV/AIDS estimates and projections 2007-2012. Report published by Family Health International 2009.

19 Curran GM, Bauer M, Mittman B, et al. Effectiveness-implementation hybrid designs: combining elements of clinical effectiveness and implementation research to enhance public health impact. Med Care 2012;50:217-26.

20 World Health Organization. Guidelines for the screening, care and treatment of persons with chronic hepatitis $\mathrm{C}$ infection_Updated version, April 2016, 2016

21 Vietnam Ministry of Health. Guidelines on diagnosis and treatment of hepatitis C. Decision N ${ }^{\circ} 5012$, September 20th 2016, 2016

22 Oppe M, Devlin NJ, van Hout B, et al. A program of methodological research to arrive at the new international EQ-5D-5L valuation protocol. Value Health 2014;17:445-53.
23 Martin NK, Hickman M, Hutchinson SJ, et al. Combination interventions to prevent HCV transmission among people who inject drugs: modeling the impact of antiviral treatment, needle and syringe programs, and opiate substitution therapy. Clin Infect Dis 2013;57 Suppl 2:S39-45.

24 Martin NK, Vickerman P, Miners A, et al. Cost-effectiveness of hepatitis $C$ virus antiviral treatment for injection drug user populations. Hepatology 2012;55:49-57.

25 Smith DJ, Combellick J, Jordan AE, et al. Hepatitis C virus (HCV) disease progression in people who inject drugs (PWID): a systematic review and meta-analysis. Int J Drug Policy 2015;26:911-21.

26 Pitt C, Vassall A, Teerawattananon Y, et al. Foreword: health economic evaluations in low- and middle-income countries: methodological issues and challenges for priority setting. Health Econ 2016;25 Suppl 1:1-5. 